\title{
First isolation of Klebsiella variicola from a horse pleural effusion
}

\author{
Elisabetta Mondo, Riccardo Rinnovati, Alessandro Spadari, Federica Giacometti ${ }^{*} \mathbb{D}$, Andrea Serraino, \\ Federica Savini and Silvia Piva
}

\begin{abstract}
Background: Respiratory diseases are the second most common cause of illnesses in horses, their etiology can be viral, bacterial, immune-mediated, or mechanical (Racklyeft and Love DN, Aust Vet J 78:549-59, 2000; Austin et al., J Am Vet Med Assoc 207:325-328, 1995; Arroyo et al., J Vet Intern Med 31:894-900, 2017). Klebsiella variicola is a Gram-negative bacterium that was initially identified as an endophyte in soil and plants such as bananas, rice, sugar cane and maize but recent studies have identified this microorganism as an emerging pathogen in humans (Rodríguez-Medina et al., Emerg Microbes Infect 8:973-988, 2019; Fontana et al., J Clin Microbiol 57:e00825-18, 2019; Rosenblueth et al., Syst Appl Microbiol 27:27-35, 2004).

This paper describes, for the first time to our knowledge, the isolation of $K$. variicola from pleural effusion in a male adult horse.

Case presentation: 17-years Italian Saddle Horse with respiratory distress and fever was admitted to the Veterinary Teaching Hospital of the Department of Veterinary Medical Sciences, University of Bologna. At home, the patient had undergone antibiotic therapy without clinical improvement. Vital signs on admission revealed an increased respiratory rate, tachycardia, pyrexia and weight loss. The animal was submitted for collateral examination including thoracic radiology and ultrasound and thoracoscopy that showed bilateral pleural effusion associated with multifocal pulmonary atelectasis.

During the thoracoscopic examination, that confirmed the presence of a seropurulent pleural effusion, a sample of pleural fluid was collected and Gram-negative bacteria were isolated and subjected to matrix-assisted laser desorption/ionization time-of-flight mass spectrometry (MALDI-TOF MS) that allowed the identification of $K$. variicola. The isolate was sensitive to amikacin, cefazolin, enrofloxacin, marbofloxacin, tetracycline, and trimethoprimsulfamethoxazole;the horse was treated with Oxytetracycline and amikacin. Despite a general health improvement of the subject, the pleural effusion did not resolve after treatment.

Conclusions: This paper describes, for the first time, the isolation of $K$. variicola in a horse with respiratory disease. The misidentification between $K$. variicola and $K$. pneumoniae has caused unawareness about significant aspects of this bacterial species. In fact, even though in animals the role of this bacterium is not clear, in humans it has been recognized as an emerging pathogen. The use of new methods for bacterial identification will probably lead to the isolation of a greater number of strains which will have to be studied to acquire knowledge that will be useful to clarify the clinical importance and relevance of $K$. variicola also in animals.
\end{abstract}

Keywords: Klebsiella variicola, Horse, Respiratory disease

\footnotetext{
*Correspondence: federica.giacometti3@unibo.it

Department of Veterinary Medical Sciences, University of Bologna, Via Tolara

di Sopra 50, Bologna 40064, Italy
}

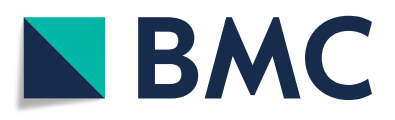

(C) The Author(s). 2021 Open Access This article is licensed under a Creative Commons Attribution 4.0 International License, which permits use, sharing, adaptation, distribution and reproduction in any medium or format, as long as you give appropriate credit to the original author(s) and the source, provide a link to the Creative Commons licence, and indicate if changes were made. The images or other third party material in this article are included in the article's Creative Commons licence, unless indicated otherwise in a credit line to the material. If material is not included in the article's Creative Commons licence and your intended use is not permitted by statutory regulation or exceeds the permitted use, you will need to obtain permission directly from the copyright holder. To view a copy of this licence, visit http://creativecommons.org/licenses/by/4.0/ The Creative Commons Public Domain Dedication waiver (http://creativecommons.org/publicdomain/zero/1.0/) applies to the data made available in this article, unless otherwise stated in a credit line to the data. 


\section{Background}

Respiratory diseases are the second most common cause of illnesses in horses, the etiology can be viral, bacterial, immune-mediated, or mechanical [1]. Pleuropneumonia is not uncommon in horses, the most common risk factors include excessive exercise, transportation and viral respiratory infections that lead to an immune suppression status [2]. Streptococcus equi subsp. zooepidemicus is the most common bacteria isolated from horses with pleuropneumonia, but many other opportunistic pathogens have been isolated in cases of pneumonia, also often caused by mixed infections between aerobic and anaerobic bacteria. Other common aerobic bacteria isolated from pleuropneumonia include Streptococcus spp., Pasteurella spp., Escherichia coli, Klebsiella spp. Actinobacillus spp., Enterobacter spp.; Bacteroides spp., Clostridium spp., Fusobacterium spp. and Peptostreptococcus spp. as anaerobes [3].

Klebsiella variicola is a Gram-negative, nitrogenfixing, nonspore-forming, nonmotile rod-shaped bacterium, firstly isolated in Mexico in 2004 and identified on the basis of total DNA-DNA hybridization and phylogenetic analysis of the sequences of $r p o B$ gene [4]. $K$. variicola was initially identified as an endophyte in soil and plants such as bananas, rice, sugar cane and maize, but recent studies identify this microorganisms as an emerging pathogen in humans; it has been isolated from many clinical samples, including blood, tracheal aspirates, several types of secretions as well as the respiratory and urinary tract [4-7]. In animals, Klebsiella spp. are associated with infections of the urinary tract, respiratory tract and sepsis [8], while $K$. variicola has to date only been described in a bovine mastitis [9].

This paper describes, for the first time to our knowledge, the isolation of $K$. variicola from pleural effusion in a male adult horse showing clinical signs associated with respiratory disease, highlighting the risk of misidentification with Klebsiella pneumoniae.

\section{Case presentation}

In February 2020, a 17-years Italian Saddle Horse male with respiratory distress and fever was admitted to the Veterinary Teaching Hospital of the Department of Veterinary Medical Sciences, University of Bologna. At home, the patient had undergone antibiotic therapy in a sequence (Penicillin G- procain 44,000 IU/KG for five days every $24 \mathrm{~h} \mathrm{IM}$; Oxitetracicline $6.6 \mathrm{mg} / \mathrm{kg}$ for 5 days every $24 \mathrm{~h} \mathrm{IV}$; Ceftiofur sodium $2.2 \mathrm{mg} / \mathrm{kg}$ IV every $12 \mathrm{~h}$ for ten days), without clinical improvement. Vital signs on admission revealed an increased respiratory rate $(>15$ breaths/min), tachycardia (heart rate $>44$ beats/min; 56/ 64 ), pyrexia $\left(>38.5^{\circ} \mathrm{C} ; 31 / 62\right)$, and weight loss $(15 / 59)$. Further collateral examination including thoracic radiology and ultrasound and thoracoscopy, were performed.
The radiographic examination, performed in three left to right to left latero-lateral views (Fig. 1), revealed a bilateral line of fluid extended across the entire chest, with an approximate height of $20 \mathrm{~cm}$ on the right side and 17 $\mathrm{cm}$ on the left side, in particular at the mediastinal level. The ultrasound examination showed bilateral pleural effusion associated with multifocal lung atelectasis. The latter was more severe in the right hemithorax where the parietal and diaphragmatic pleura were also involved (Fig. 2). The thoracoscopic examination confirmed the presence of a seropurulent pleural effusion, but no superficial obvious lesions to the lung and to the parietal and visceral pleura were evidenced. Along with the first trocar insertion, a sterile sample of turbid yellow thoracic fluid was collected and sent to the laboratory for cytological and bacteriological exams. Cell count was performed with ADVIA 2120 and resulted in 43'000 eritrocytes/ $\mu \mathrm{l}$, 91'267 total nucleated cells / $\mu \mathrm{l}$ with $92 \%$ non-degenerated or picnotic neutropils, $4 \%$ activated

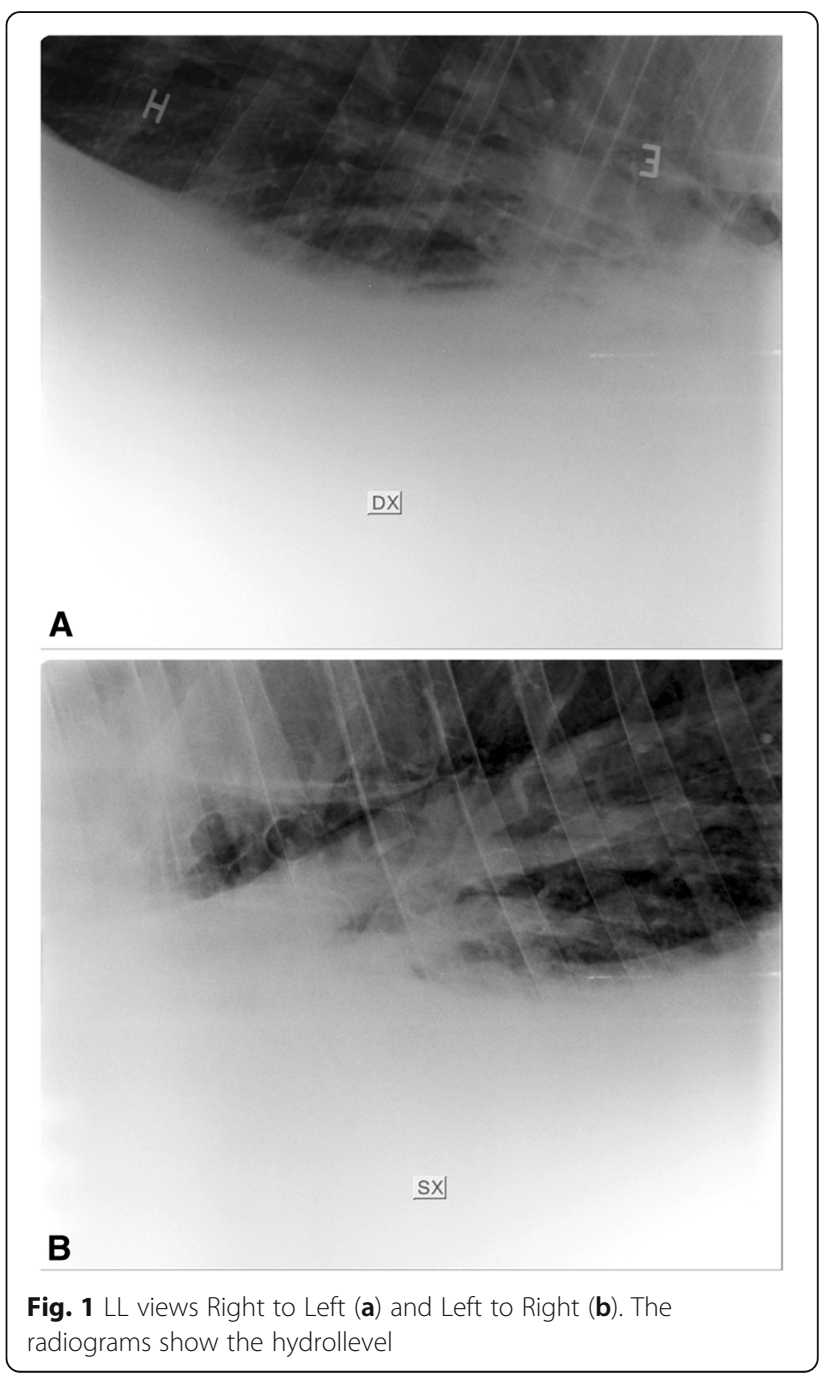




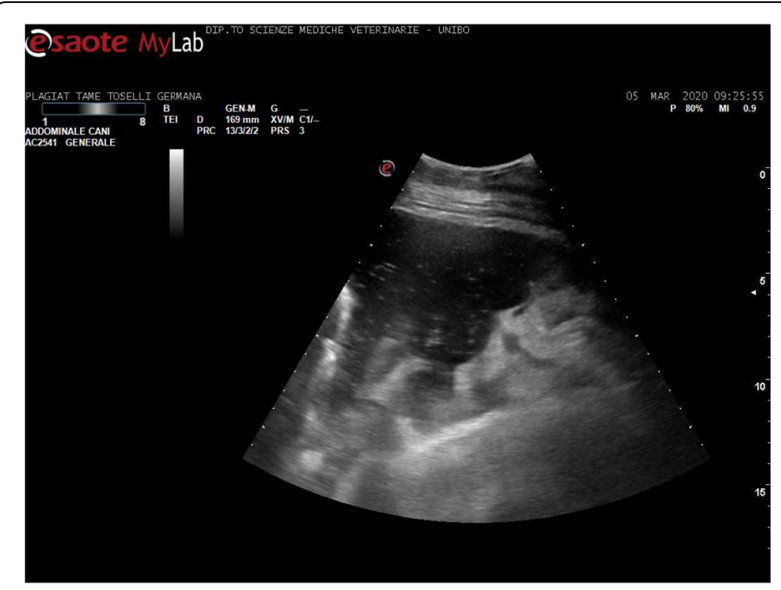

Fig. 2 Ultrasound image of pleural space with pleural effusion and pleural fluid

macrophages and $4 \%$ small lymphocytes. Neither intra nor extracellular microorganisms were observed.

The haematology did not reveal anything particularly significant.

Under thoracoscopic guidance a second opening for drainage tube insertion was created at the 9th right intercostal space $10 \mathrm{~cm}$ dorsal to a horizontal line passing by the olecranic tuberosity. A drainage tube of $28 \mathrm{~F}$ was inserted for daily evacuation of the fluid and pleural wash.

A sample of pleural fluid collected during thoracoscopy was collected for routine aerobic and anaerobic bacterial culture. After $24 \mathrm{~h}$ of incubation, round and mucoid colonies were isolated on Blood Agar Base with $5 \%$ horse blood and on MacConkey agar plates. Microscopically, colonies appeared as Gram-negative rodshaped (Fig. 3) and, by biochemical tests, they were catalase-positive and oxidase-negative. An isolate from Blood Agar was subcultured on Tryptone Soya Agar and incubated for $24 \mathrm{~h}$ at $37 \pm 1{ }^{\circ} \mathrm{C}$ in aerobic condition. Subsequent identification was performed using matrixassisted laser desorption/ionization time-of-flight mass spectrometry (MALDI-TOF MS) (MALDI biotyper, Bruker Inc., USA) instrument: $K$. variicola was identified at species level with a score of 2,25 using the BRUKER BIOTYPER version 3.0 software. An antimicrobial susceptibility test was performed by means of the disc diffusion method according to Clinical and Laboratory Standards Institute (CLSI) guidelines $[10,11]$ by using antimicrobial discs. The isolate was sensitive to amikacin $(30 \mu \mathrm{g})$, amoxicillin- clavulanate $(30 \mu \mathrm{g})$, chloramphenicol $(30 \mu \mathrm{g})$, cefazolin $(30 \mu \mathrm{g})$, enrofloxacin $(5 \mu \mathrm{g})$, marbofloxacin $(5 \mu \mathrm{g})$, tetracycline $(30 \mu \mathrm{g})$, and trimethoprimsulfamethoxazole $(1.25 / 23.7 \mu \mathrm{g})$, Intermediate sensitivity

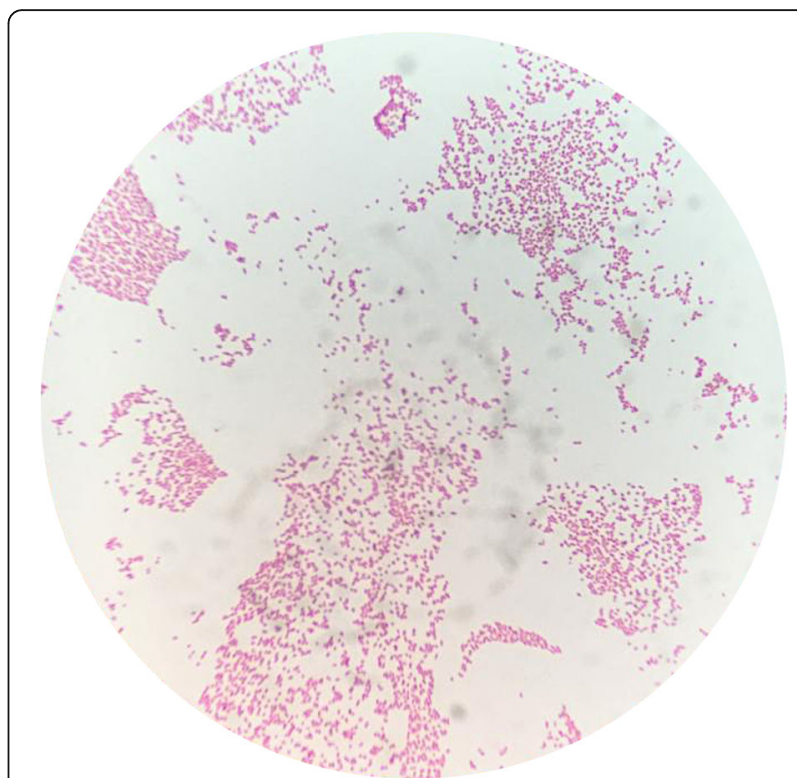

Fig. 3 Gram stain of Klebsiella variicola. The Gram stain of a positive culture demonstrates Gram-negative bacilli on microscopy (x100)

was evidenced to gentamicin $(10 \mu \mathrm{g})$, streptomycin (10 $\mu \mathrm{g})$, ceftiofur $(30 \mu \mathrm{g})$, while the isolates was resistant to ampicillin $(10 \mu \mathrm{g})$, and sulfonamides $(300 \mu \mathrm{g})$.

A combination disc test following the instructions reported in EUCAST (2017) for the evaluation of ESBL was performed (Oxoid, Basinstoke, UK) media and antimicrobial discs. The isolate was tested for ceftazidime alone and in combination with clavulanic acid using Kirby Bauer disk agar diffusion method [12].

The inhibition zones of the cephalosporins alone, as well as in combination with clavulanic acid were compared. The test was considered positive when an increase $\geq 5 \mathrm{~mm}$ in zone of inhibition was observed in the presence of clavulanic acid, compared with the cephalosporin alone but in our isolate the $\geq 5 \mathrm{~mm}$ increase was not observed. Oxoid (Basinstoke, UK) media and antimicrobial discs were used.

According to the results, the horse was treated I.V. with Oxytetracycline $(6,6 \mathrm{mg} / \mathrm{kg}$ sid) for 10 consecutive days. In addition, a daily intrathoracic lavage with amikacin (400 gr) diluted in 2 liters of saline was administered. The general health of the horse improved after 10 weeks from hospital discharge but the pleural effusion was not resolved.

\section{Discussion and conclusions}

In this paper, isolation of $K$. variicola from pleural effusion in a male adult horse with respiratory symptoms is reported. Although a single isolation from a unique pleural effusion sample has been performed, $K$. variicola grew in a monomicrobic growth. In humans, $K$. variicola 
is considered an emerging pathogen, while in animals its role is still unclear. The study carried out by Maatallah et al. (2014) reported that human bacteremia infections caused by $K$. variicola have higher 30 -day mortality rates than those of infections caused by K. pneumoniae, suggesting the higher virulence of $K$. variicola [13]. Moreover, several researches estimate that around $10 \%$ of $K$. pneumoniae isolated in human infections are misidentifications of $K$. variicola [6]. In literature, $K$. variicola has rarely been isolated in animals [8], in particular, among food-producing animals Podder and Colleagues (2014) evidenced that an isolate of $K$. variicola can cause clinical mastisis in dairy cattle, as it is normally found in soil and feed, and not in milk from infected animals [9].

Biochemical and phenotypic features' overlapping between $K$. pneumoniae and $K$. variicola make identification of the latter difficult by traditional microbiological methods [14], leading to underestimate its pathological importance.

Furthermore, since the sequences of $K$. variicola, and K. quasipneumoniae, have been included in common molecular databases only recently, the two species have been either unidentified or misidentified. Misidentification has caused unawareness about significant aspects of this bacterial species, that is becoming a public health concern not only for the infections that it can cause but also due to its potential to acquire antimicrobial and virulence genes, hampering the clinical management of the provoked infections [15].

The available methods differentiating and identifying bacterial subspecies within the K. pneumoniae complex, such as $K$. variicola, were only based on genomic and phylogenetic analysis, until a few years ago. In particular, genomic analysis is based on PCR amplification of chromosomal $\beta$-lactamases, multiplex PCR, or PCR for the research of $y g g E$ gene, while phylogenetic analysis is based on the most commonly used gene rpoB $[4,16]$.

In 2018, $K$. variicola was added to the MALDI-TOF MS Bruker reference library, and shortly after a study demonstrated $100 \%$ sensitivity and specificity [14] of this methodology in differentiating among $K$. pneumoniae, $K$. quasipneumoniae, and $K$. variicola. Hence, the use of MALDI- TOF for bacterial identification in routine microbiology is preferable to conventional phenotypic/biochemical techniques in terms of speed and precision. Nevertheless, PCR and phylogenetic analysis represent a valid alternative when MALDI-TOF technology is not available.

Although successfully applied for the identification of human pathogens, so far its use for routine identification of veterinary bacterial isolate is still limited [17].

Correct identification of $K$. variicola is of the utmost importance because this new bacterium may be a pathogen agent in the animals like it is classified as an important human pathogen. Correct identification of the respiratory infection causative agent is the first step for the right administration of a specific antimicrobial therapy, and it is fundamental for the animal's healing and the prudent use of antibiotics. In line with our findings, $K$. variicola isolates are broadly antimicrobial susceptible (even if reports of ESBL-producing and carbapenemaseproducing isolates have increased) [5] and have lower antibiotic resistance rates than other Klebsiella species. Nevertheless lower antibiotic resistance rates do not necessarily correlate with better treatment outcomes in $K$. variicola infections [18]. In addition, the genome of $K$. variicola is considered an open genome, namely, the microorganism is able to incorporate genes that allow its adaptation to different environments by conferring resistance to antibiotics to which it was previously susceptible, as well as expanding its pathogenicity by incorporating virulence factors [19].

Further efforts should be performed to differentiate $K$. variicola from $K$. pneumoniae complex since adequate identification of $K$. variicola is not routinely performed in clinical specimens and its real incidence is unknown. The use of new methods for bacterial identification will probably lead to the isolation of a greater number of strains that will widen knowledge on thei pathogenic power and diffusion, as well as on the clinical importance and relevance of $K$. variicola in human and animal infection.

\section{Conclusion}

The present study describes the isolation of $K$. variicola in a horse with respiratory disease. In the past, misidentification with $K$. pneumoniae has caused unawareness about significant aspects of this bacterial species, as for example its possible role in animals' diseases. In fact, probably due to the poor isolation rate its role is not clear in animals, while it has been demonstrated, to be an emerging human pathogen. Further studies will be needed to understand the spread and the virulence pattern of $K$. variicola in veterinary medicine.

\section{Abbreviations \\ MALDI-TOF MS: Matrix-assisted laser desorption/ionization time-of-flight mass spectrometry; CLSI: Clinical and Laboratory Standards Institute; EUCAST: European Committee on Antimicrobial Susceptibility Testing; ESBL: Extended Spectrum-Beta-Lactamase}

\section{Acknowledgements}

Not applicable.

\section{Authors' contributions}

EM has made substantial contribution to the concept and design of the work, to the microbiological analysis and has drafted the work. FG and AS have performed microbiological analysis and revised the work. RR and AS have performed clinical analysis and drafted the work. FS has substantively revised the work for the language. SP has made substantial contribution to the concept and design of the work, to the microbiological analysis and revised the work. All authors have approved the submitted version and any 
substantially modified version that involves the author's contribution to the study. All authors have agreed both to be personally accountable for the author's own contributions and to ensure that questions related to the accuracy or integrity of any part of the work, even ones in which the author was not personally involved, are appropriately investigated, resolved, and the resolution documented in the literature.

\section{Funding}

This manuscript has not received any funding.

\section{Availability of data and materials}

The datasets used and/or analyzed during the current study are available from the corresponding author on reasonable request.

\section{Ethics approval and consent to participate}

We do not have an ethical statement because all the information is collected during the routine diagnostic procedures. The owner was informed about data publication.

\section{Consent for publication}

The owner of the horse gave written consent for publication.

\section{Competing interests}

The authors declare that they have no competing interests.

Received: 4 August 2020 Accepted: 26 January 2021

Published online: 12 February 2021

\section{References}

1. Racklyeft DJ, Love DN. Bacterial infection of the lower respiratory tract in 34 horses. Aust Vet J. 2000;78(8):549-59. https://doi.org/10.1111/j.1751-0813. 2000.tb11901.x.

2. Austin SM, Foreman JH, Hungerford LL. Case-control study of risk factors for development of pleuropneumonia in horses. J Am Vet Med Assoc. 1995; 207:325-8.

3. Arroyo MG, Slovis NM, Moore GE, Taylor SD. Factors associated with survival in 97 horses with septic pleuropneumonia. J Vet Intern Med. 2017:31:894900 .

4. Rosenblueth M, Martínez L, Silva J, Martínez-Romero E. Klebsiella variicola, a novel species with clinical and plant-associated isolates. Syst Appl Microbiol. 2004;27(1):27-35. https://doi.org/10.1078/0723-2020-00261.

5. Rodríguez-Medina N, Barrios-Camacho H, Duran-Bedolla J, Garza-Ramos U. Klebsiella variicola: an emerging pathogen in humans. Emerg Microbes Infect. 2019:8(1):973-88. https://doi.org/10.1080/22221751.2019.1634981.

6. Fontana L, Bonura E, Lyski Z, Messer W. The brief case: Klebsiella variicolaidentifying the misidentified. J Clin Microbiol. 2019;57(1):e00825-18. https:// doi.org/10.1128/JCM.00826-18.

7. Imai K, Ishibashi N, Kodana M, Tarumoto N, Sakai J, Kawamura T, Takeuchi S, Taji Y, Ebihara Y, Ikebuchi K, Murakami T, Maeda T, Mitsutake K, Maesaki S. Clinical characteristics in blood stream infections caused by Klebsiella pneumoniae, Klebsiella variicola, and Klebsiella quasipneumoniae: a comparative study, Japan, 2014-2017. BMC Infect Dis. 2019;19(1):946. https://doi.org/10.1186/s12879-019-4498-x.

8. Brisse $S$, van Duijkeren E. Identification and antimicrobial susceptibility of 100 Klebsiella animal clinical isolates. Vet Microbiol. 2005:105(3-4):307-12. https://doi.org/10.1016/j.vetmic.2004.11.010.

9. Podder MP, Rogers L, Daley PK, Keefe GP, Whitney HG, Tahlan K. Klebsiella species associated with bovine mastitis in Newfoundland. PLoS One. 2014 9(9):e106518. https://doi.org/10.1371/journal.pone.0106518.

10. CLSI. Performance Standards for Antimicrobial Disk and Dilution Susceptibility Tests for Bacteria Isolated From Animals, 4th Edition. Wayne: Clinical and Laboratory Standards Institute. (Document VET08, ED4:2018).

11. CLSI. Performance Standards for Antimicrobial Disk and Dilution Susceptibility Tests for Bacteria Isolated From Animals, 3th Edition. Wayne: Clinical and Laboratory Standards Institute. (Document VET01, ED3:2015).

12. EUCAST. Guidelines for detection of resistance mechanisms and specific resistances of clinical and/ or epidemiological importance. version 2.01. 2017.

13. Maatallah $M$, Vading $M$, Kabir $M H$, Bakhrouf $A$, Kalin $M$, Nauclér P, Brisse $S$, Giske CG. Klebsiella variicola is a frequent cause of bloodstream infection in the Stockholm area, and associated with higher mortality compared to $K$. pneumoniae. PLoS One. 2014;9(11):e113539. https://doi.org/10.1371/journal pone.0113539.

14. Rodrigues C, Passet V, Rakotondrasoa A, Brisse S. Identification of Klebsiella pneumoniae, Klebsiella quasipneumoniae, Klebsiella variicola and related phylogroups by MALDI-TOF mass spectrometry. Front Microbiol. 2018;9: 3000. https://doi.org/10.3389/fmicb.2018.03000.

15. Jiang SF, Liu Y, Xiao MY, Ruan CJ, Lu ZJ. Draft genome sequence of Klebsiella variicola strain KV321 isolated from rhizosphere soil of Pisolithus tinctorius-Eucalyptus Mycorrhiza. Genome Announc

16. Garza-Ramos U, Silva-Sánchez J, Martínez-Romero E, Tinoco P, Pina-Gonzales $\mathrm{M}$, Barrios $\mathrm{H}$, et al. Development of a Multiplex-PCR probe system for the proper identification of Klebsiella variicola microbial genetics, genomics and proteomics. BMC Microbiol. 2015;13:64. https://doi.org/10.1186/s12866-0150396-6.

17. Carbonnelle E, Mesquita C, Bille E, Day N, Dauphin B, Beretti JL, Ferroni A, Gutmann L, Nassif X. MALDI-TOF mass spectrometry tools for bacterial identification in clinical microbiology laboratory. Clin Biochem. 2014;44(1): 104-9. https://doi.org/10.1016/j.clinbiochem.2010.06.017.

18. Berry GJ, Loeffelholz MJ, Williams-Bouyer N. An investigation into laboratory misidentification of a bloodstream Klebsiella variicola infection. J Clin Microbiol. 2015;53:2793-4. https://doi.org/10.1128/JCM.00841-15.

19. Holt KE, Wertheim H, Zadoks RN, Baker S, Whitehouse CA, Dance D, Jenney A, Connor TR, Hsu LY, Severin J, Brisse S, Cao H, Wilksch J, Gorrie C, Schultz MB, Edwards DJ, Nguyen KV, Nguyen TV, Dao TT, Mensink M, Minh VL, Nhu NTK, Schultsz C, Kuntaman K, Newton PN, Moore CE, Strugnell RA, Thomson NR. Genomic analysis of diversity, population structure, virulence, and antimicrobial resistance in Klebsiella pneumoniae, an urgent threat to public health. Proc Natl Acad Sci USA. 2015;112:E3574-81. https://doi.org/10.1073/ pnas.1501049112

\section{Publisher's Note}

Springer Nature remains neutral with regard to jurisdictional claims in published maps and institutional affiliations.
Ready to submit your research? Choose BMC and benefit from:

- fast, convenient online submission

- thorough peer review by experienced researchers in your field

- rapid publication on acceptance

- support for research data, including large and complex data types

- gold Open Access which fosters wider collaboration and increased citations

- maximum visibility for your research: over $100 \mathrm{M}$ website views per year

At BMC, research is always in progress.

Learn more biomedcentral.com/submissions 